gle's medium (DMEM), then treated with serum- and phosphate-free DMEM containing $100 \mu \mathrm{M}$ ascorbate with or without ISO (10 $\mu \mathrm{M}$ final concentration) and incubated at $37^{\circ} \mathrm{C}$ for $15 \mathrm{~min}$. The cells were then assayed for whole-cell phosphorylation as described (7). The extent of receptor phosphorylation was quantitated with a Molecular Dynamics phosphorimaging system and ImageQuant software.
23. We thank R.J. Lefkowitz for encouragement and insightful reading of the manuscript and members of his laboratory for antisera to $\beta A R K 1$ and -2 , antisera to $\beta$-arrestin-1 and -2 , wild-type $\beta A R K 1, \beta$-arrestin-1 and -2 cDNA constructs, and wild-type and phosphorylation site-deficient $\beta_{2} A R$ cDNA constructs. Supported in part by a grant from the $\mathrm{Na}$ tional Institutes of Health (NS 19576) and a Bristol
Myers Squibb unrestricted grant award to M.G.C. S.S.G.F. is a fellow of the Medical Research Council of Canada, W.E.D. III is a recipient of a Howard Hughes Medical Institute research training fellowship for medical students, and L.S.B. is a recipient of a Howard Hughes postdoctoral fellowship.

3 October 1995; accepted 20 November 1995

\title{
The Effect of Social Experience on Serotonergic Modulation of the Escape Circuit of Crayfish
}

\author{
Shih-Rung Yeh, Russell A. Fricke, ${ }^{\star}$ Donald H. Edwards $\dagger$
}

The neuromodulator serotonin has widespread effects in the nervous systems of many animals, often influencing aggression and dominance status. In crayfish, the effect of serotonin on the neural circuit for tailflip escape behavior was found to depend on the animal's social experience. Serotonin reversibly enhanced the response to sensory stimuli of the lateral giant (LG) tailflip command neuron in socially dominant crayfish, reversibly inhibited it in subordinate animals, and persistently enhanced it in socially isolated crayfish. Serotonin receptor agonists had opposing effects: A vertebrate serotonin type 1 receptor agonist inhibited the LG neurons in dominant and subordinate crayfish and had no effect in isolates, whereas a vertebrate serotonin type 2 receptor agonist enhanced the LG neurons' responses in all three types of crayfish. The LG neurons appear to have at least two populations of serotonin receptors that differ in efficacy in dominant, subordinate, and socially isolate crayfish.

Serotonin is a neuromodulator involved in the expression of dominance and aggression in many animals, including humans (1). In lobsters and crayfish, serotonin injected into the circulatory system causes them to adopt an elevated, flexed ("dominant") posture (2). Serotonin-containing neurons function as postural gain-setting elements, biased toward flexion, that enhance the responsiveness of the motor circuitry to coordinated postural commands (3).

Serotonin plays a similar gain-setting role in the neural circuit for tailflip escape in crayfish (4); however, we found that the modulatory effect of serotonin depends on the social experience of the animal. The LG neuron is a command neuron for tailflip that is excited by mechanosensory input from the abdomen (Fig. 1) (5). It has been shown that serotonin applied through the arterial blood supply inhibits both the monosynaptic $(\alpha)$ and disynaptic $(\beta)$ components of the compound excitatory postsynaptic potential (EPSP) evoked in the LG neuron by sensory nerve stimulation $(4,6)$. Serotonin also depolarized the LG neuron by 2 to $3 \mathrm{mV}$ and caused a drop in the input resistance of the neuron's distal dendrites, suggesting that serotonin's

S.-R. Yeh and D. H. Edwards, Department of Biology, Georgia State University, Atlanta, GA 30302-4010, USA. R. A. Fricke, Department of Anatomy and Cell Biology, Emory University, Atlanta, GA 30322, USA

"Present address: Office of the Commissioner, Schenectady County Public Health Services, One Broadway Center, Suite 840, Schenectady, NY 12305, USA.

tTo whom correspondence should be addressed. effects were mediated on the LG neuron itself. Serotonin-containing neurons and fibers with varicose terminals are present in each abdominal ganglion, but the relation between LG dendrites and the terminals of serotonergic neurons has not been described (7). Endogenous serotonin could affect the LG neuron's response through local ganglionic release or through neurohumoral release mechanisms.

When placed together in a small aquarium, a pair of previously isolated crayfish of similar size will interact agonistically to determine which animal is dominant and which is subordinate. The interactions usually take less than 0.5 hour, after which the subordinate moves by retreating or tailflipping to avoid contact with the dominant animal. We used these easily recognizable behavior patterns to determine the dominance status of animals paired for periods of 12 days or longer (8). We then determined the effect of bath-applied serotonin on the LG neuron's response to sensory nerve stimulation in socially isolated animals and in animals of known dominance status (9). In socially isolated animals, serotonin (50 and $100 \mu \mathrm{M}$ ) enhanced the LG neuron's responses to sensory nerve shock (Fig. 2A, left). The $\alpha$ and $\beta$ EPSPs evoked in the LG neuron by all subthreshold levels of sensory nerve shock were increased, and the stimulus threshold was reduced (10). These effects were not readily reversible and persisted after 5 hours of wash. In subordinate crayfish, however, serotonin reversibly reduced the LG neuron's EPSPs (Fig. 2A, middle): $\alpha$ and $\beta$ EPSPs evoked by all subthreshold levels of sensory nerve shock were reduced in the presence of bath-applied serotonin, and the stimulus threshold of an LG spike was increased (10). A 1-hour wash with saline restored the LG EPSPs and in some instances produced a rebound excitation. In dominant crayfish, serotonin reversibly enhanced LG responses over the complete range of subthreshold stimuli (Fig. 2A, right), and reduced the LG neuron's stimulus threshold (10). We obtained similar results in 37 juvenile (Fig. 3A) and 23 adult (10) crayfish Serotonin had no obvious effect on the re्त sponses of other mechanosensory interney rons, some of which contribute to the $\beta$ EPSP in the LG neuron (Fig. 1) (11). Wह conclude that serotonin's modulatory effect on the LG neuron's response depends on the्छ animal's social experience.

To determine if the different effects o serotonin on the LG neuron in dominantơ subordinate, and socially isolated crayfish were produced by different serotonin recepe tors, we repeated the experiments described above with a vertebrate serotonin type $\left(5-\mathrm{HT}_{1}\right)$ or serotonin type $2\left(5-\mathrm{HT}_{2}\right)$ recep tor agonist substituted for serotonin. Al though the vertebrate $5-\mathrm{HT}_{1}$ agonist $1-\left(3 \frac{3}{\varepsilon}\right.$ chlorophenyl)piperazine dihydrochloride (m-CPP $\mathrm{Cl}_{2}$ ) had no effect on the L neuron's responses in the social isolates, id reduced the $\alpha$ and $\beta$ LG EPSPs evoked bxo the entire range of subthreshold stimuli ire all dominant and subordinate crayfish (Fig $2 \mathrm{~B}$ and Fig. 3B), and it raised the stimuluo

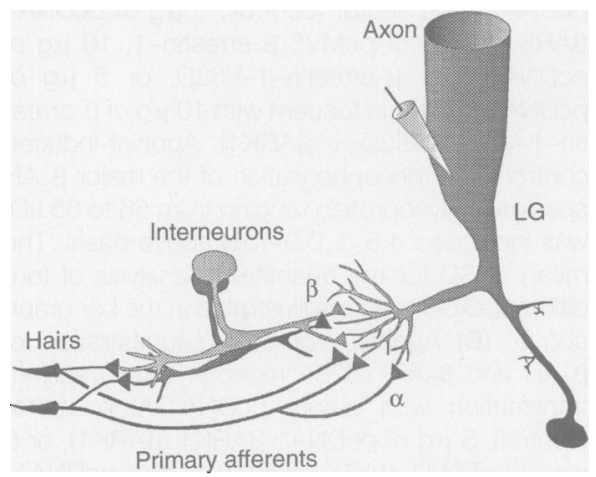

Fig. 1. Afferent portion of the LG tailflip circuit, showing monosynaptic $(\alpha)$ and disynaptic $(\beta)$ afferent paths from abdominal hairs to the LG neuron (5). A single spike in the $L G$ is sufficient to trigger tailflip escape movements. Afferent nerves from the sensory hairs were stimulated in the presence of serotonin and 5-HT agonists; LG EPSPs were recorded from the proximal axon (9). 
threshold for a spike in the LG neuron of both animals (10). These effects were largely reversed by a 1 -hour saline wash. A vertebrate 5-HT, agonist, $\alpha$-methylserotonin maleate ( $\alpha-\mathrm{CH}_{3}$ 5-HT maleate), enhanced the LG neurons' EPSPs (Fig. $2 \mathrm{C}$ and Fig. 3C) and reduced the cells' stimulus thresholds (10) in isolate, dominant, and subordinate crayfish. The effects of the $5-\mathrm{HT}_{2}$ agonist persisted after more than 2 hours of saline perfusion in the isolates, but were removed by 1 hour of saline perfusion in the dominants and subordinates. Similar results were obtained with adult $(>8 \mathrm{~cm})$ crayfish (10).

We conclude that at least two and perhaps three types of 5-HT receptor can modulate the LG neuron's response in crayfish: (i) a 5-HT - like receptor that produces response inhibition only while serotonin is present, (ii) a 5-HT, -like receptor that produces response enhancement only while serotonin is present, and possibly (iii) a 5-HT - -like receptor that produces response enhancement that persists after serotonin has been washed away. The short-term inhibitory and excitatory receptors (types i and ii) are both either masked or missing from isolates but are present in dominant and subordinate crayfish. The long-term response enhancement seen only in isolate crayfish could be produced by a separate type iii receptor or it might result from modification of the type ii receptor mechanism.

Differential activation of the two shortterm receptors may account for the opposing effects of serotonin on the LG neuron's response in dominant and subordinate crayfish. The effects of the $5-\mathrm{HT}_{1}-$ like receptor appear to predominate in subordinate crayfish where serotonin is inhibitory, whereas the effects of the short-term $5-\mathrm{HT}_{2}$-like receptors appear to predominate in the LGs of dominant crayfish where serotonin is excitatory. Measurement of the dose-response functions of the LG neuron to specific agonists of each receptor type is necessary to establish this point quantitatively.

To determine the time course of the change in serotonin's effect on the LG after a change in social status, we tested the effect of serotonin on the LG neuron's responses in new subordinate and dominant animals that had been paired for periods from 1 to 12 days after 1 or more months of isolation. The enhancing effect of serotonin on the LG neuron's EPSPs seen in the isolate crayfish (Fig. 2A, Fig. 3A, Fig. 4;

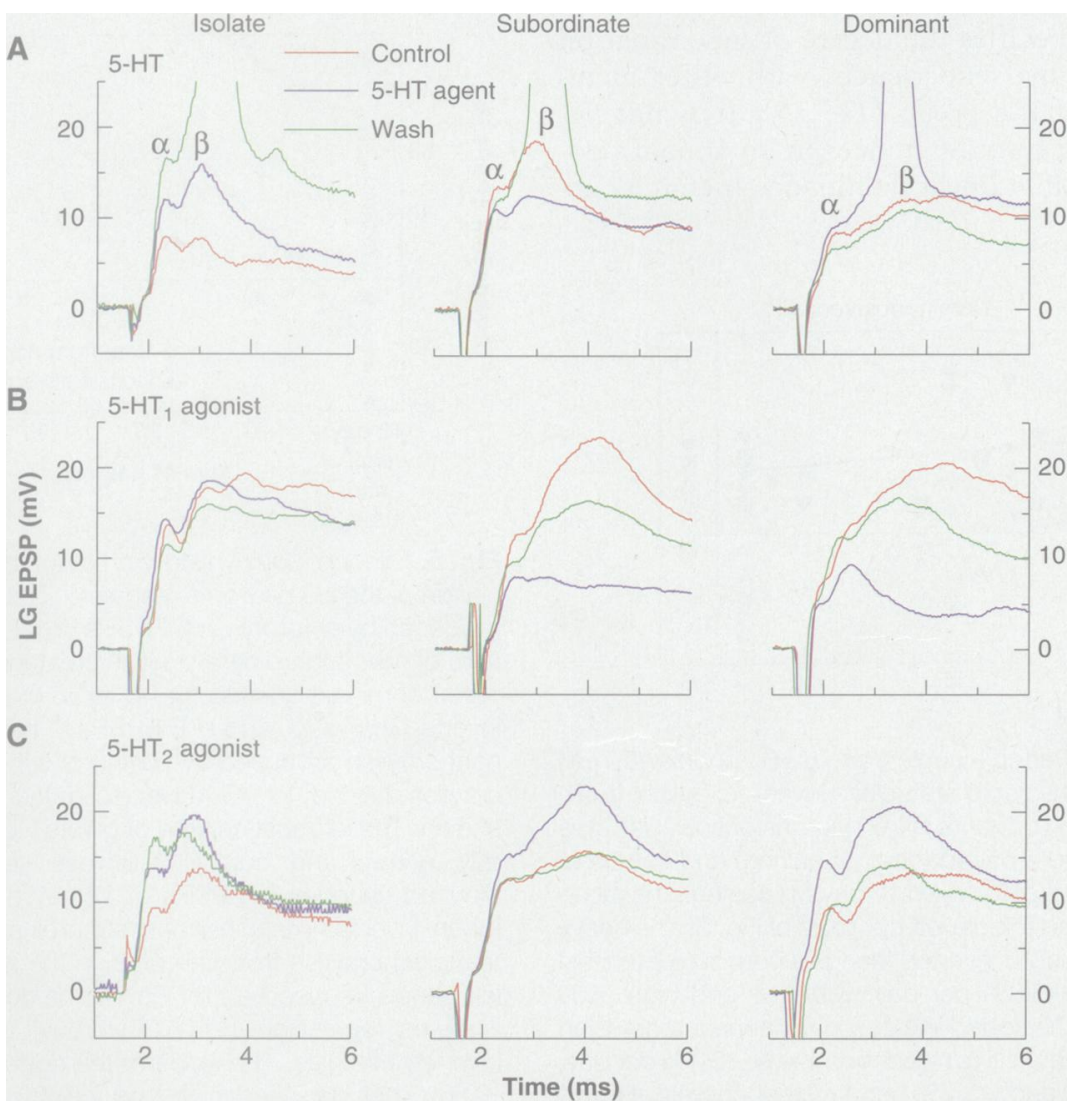

Fig. 2. Different effects of $50 \mu \mathrm{M}$ concentrations of (A) serotonin (5-HT); (B) a 5- $\mathrm{HT}_{1}$ agonist, $\mathrm{m}-\mathrm{CPP} \mathrm{Cl}_{2}$; and (C) a $5-\mathrm{HT}_{2}$ agonist, $\alpha-\mathrm{CH}_{3} 5-\mathrm{HT}$ maleate, on $\mathrm{LG}$ responses in socially isolated (left column), subordinate (middle column), and dominant (right column) juvenile crayfish. Responses of nine crayfish are shown; in all experiments, each crayfish was exposed either to 5-HT or a single 5-HT agonist. Monosynaptic $(\alpha)$ and disynaptic ( $\beta$ ) LG EPSPs were evoked by sensory nerve stimulation before (control), during, and after (wash) application of each 5-HT agent. "isolate" in left and right panels) decreased linearly with time in the new subordinate animals and reversed after 7 days of pairing to become increasingly inhibitory (Fig. 4 , left panel). The inhibitory effect of serotonin was reversed after reisolation of the subordinate crayfish for more than 8 days (Fig. 4, "reisolate") (12). In the new dominant crayfish, the LG neurons' responses continued to be enhanced by serotonin over 12 days of pairing (Fig. 4, right).

Changes in the LG neuron's responsiveness to the $5-\mathrm{HT}_{1}$ agonist $\mathrm{m}$-CPP $\mathrm{Cl}_{2}$ produced by a change in social status followed a time course similar to that of the response to serotonin in new subordinates. The inhibitory effect of the $5-\mathrm{HT}_{1}$ agonist increased linearly from null in the isolates to strong inhibition after 12 days of subordinate status (Fig. 4, left). In new dominant crayfish, 5-HT, agonists had little effect during the first few days after pairing but then became inhibitory as the pairing continued (Fig. 4, right). Reisolation for 10 days removed the inhibitory effects of the 5-HT, agonist (13).

These results show that changes in the set of 5-HT receptors that affect the LG

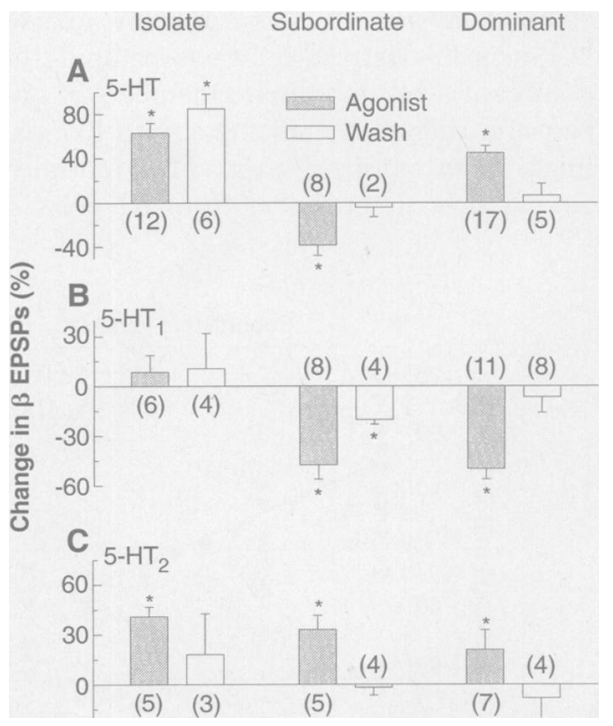

Fig. 3. Summary effects of 5-HT agonists on LG EPSPs evoked by sensory nerve stimulation. Mean (and SEM) of percent of changes (relative to control) of $\beta$ EPSPs in LGs of isolate, subordinate and dominant juvenile crayfish produced by bath application of $50 \mu \mathrm{M}$ and $100 \mu \mathrm{M}$ serotonin (5HT) (A), by $50 \mu \mathrm{M} 5-\mathrm{HT}_{1}$ agonist (B), and by 50 $\mu \mathrm{M} 5-\mathrm{HT}_{2}$ agonist (C). Effects on $\alpha$ EPSPs (10) were similar. Numbers of animals are indicated in parentheses. "Wash" responses were recorded 1 hour after saline perfusion replaced 5-HT agonist perfusion. Response changes that are significantly different than zero are indicated with an asterisk. The differences are significant at $P<$ 0.05 , as indicated by the fact that the difference between the absolute value of the response mean and the absolute value of the $95 \%$ confidence limit is greater than zero. 
neuron's response occur shortly after changes in the crayfish's social status. The inhibitory effect of $5-\mathrm{HT}_{1}$ agonists in the new subordinates develops before the reversal of serotonin's effect on the LG neuron's response. We suggest that this reversal is produced by the advent of the 5-HT - -like receptors, as well as the simultaneous decline or disappearance of the $5-\mathrm{HT}_{2}-$ like receptors that produce a long-lasting enhancement of the LG neuron's response and their replacement by the $5-\mathrm{HT}_{2}$-like receptors that produce the short-term response enhancement. A similar change occurs in the new dominant crayfish, where the excitatory effect of the short-term $5-\mathrm{HT}_{2}$-like receptors outweighs the inhibitory effect of the new $5-\mathrm{HT}_{1}$-like receptors.

The reversal of the effect of serotonin and the $5-\mathrm{HT}_{1}$ agonist after reisolation of the subordinate crayfish indicates that the set of 5-HT receptors characteristic of subordinates must be maintained by persistent sensory stimuli that confirm the animal's social status. The internal signal that links the animal's perception of its social status to the abdominal 5-HT receptors is unknown.

We wished to determine whether social dominant-to-subordinate transitions, and the reverse, could lead to corresponding changes in the effect of serotonin on the LG neuron's response. We separated the dominant and subordinate members of one pair and then re-paired them with like animals from another pair. The mortality among new subordinates from 63 pairs of social dominants was very high: Only 16 (27\%) new subordinates survived after 5 days of pairing (the others were eaten by the new dominants). The mortality among new dominants from 21 pairs of social subordinates was lower: Ten (48\%) survived. These survival rates are lower than the $65 \%$ survival rate of new subordinates in 131 pairs of former social isolates.

Unlike its action in subordinates created by pairing of isolate crayfish, serotonin continued to enhance the LG neuron's responses in subordinates created by pairing of dominant crayfish, even after 38 days of pairing (Fig. 5). This result shows that the modulatory effect of serotonin can depend not only on the crayfish's current social status (subordinate), but also on its prior status (isolate or dominant). Conversely, as in dominants created by pairing of isolates, serotonin enhanced the responses of LG neurons in dominants created by pairing of subordinates, as recorded 12 days after pairing (Fig. 5).

These results suggest that agonistic interactions can lead to different subordinate states depending on the social experience of the animal. Different subordinate states are also apparent in a linear dominance hierarchy (such as those formed by crowded crayfish), where social rank specifies the degree of subordination an animal experiences with other members of the group $(14,15)$. It is not yet known how differences in an animal's position in a linear dominance hierarchy are

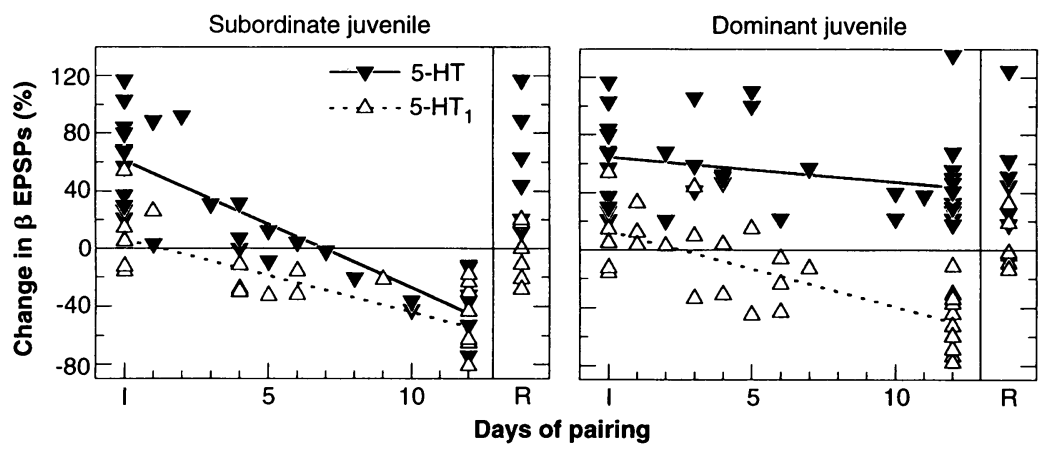

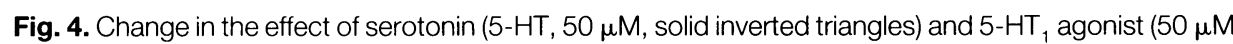
$\mathrm{m}-\mathrm{CPP} \mathrm{Cl} \mathrm{Cl}_{2}$, open triangles) on LG $\beta$ EPSPs after a change in the social status of a juvenile crayfish. (Left) Percent change from control values of $\beta$ EPSPs caused by 5 -HT agents in LG neurons of social isolates (I), in LGs of new subordinates 1 to 12 days after isolates were paired (days of pairing) and in LGs of reisolated $(R)$ subordinates recorded after 8 days of reisolation that followed 12 days of pairing. The mean and SEM of the isolate responses are given in the upper left and middle left bar plots of Fig. 3 . The heavy solid line describes a linear regression $\left(r^{2}=0.72\right)$ through the solid inverted triangles from zero (isolates) to 12 days of pairing. The slope of the regression line is $-8.8 \%$ per day, with low and high $95 \%$ confidence limits of -10.8 and $-6.9 \%$ per day, respectively. The dotted line describes a linear regression $\left(r^{2}=0.58\right)$ for the open triangles over the same period. The slope of this regression line is $-5.1 \%$ per day; the low and high $95 \%$ confidence limits of that slope are -6.9 and $-3.2 \%$ per day, respectively. (Right) Same isolate data as in the left panel (I), with corresponding data for changes in $\beta$ EPSPs of LGs of new dominants (days of pairing) and of LGs in reisolated dominants $(R)$. The regression lines are plotted as in the left panel. The $r^{2}$ value for the heavy solid line is 0.09 , and the slope of that line is $-1.7 \%$ per day, with low and high $95 \%$ confidence limits of the slope equal to -3.2 and $0.1 \%$ per day, respectively. Corresponding values for the dotted line are $r^{2}=0.57,-5.3 \%$ per day (slope), and -7.0 and $-3.57 \%$ per day (low and high 95\% confidence limits of the slope, respectively).

reflected in the physiology of the nervous system.

It is unclear whether the effects of the $5-\mathrm{HT}_{1}$ and $5-\mathrm{HT}_{2}$ agonists are mediated by receptors that are similar to $5-\mathrm{HT}_{1}$ and $5-\mathrm{HT}_{2}$ receptors of vertebrates. The $5-\mathrm{HT}_{1}$ agonist that we used, $\mathrm{mCPP} \mathrm{Cl}_{2}$, is regarded as a $5-\mathrm{HT}_{\mathrm{IB} / \mathrm{IC}}$ agonist that also has some effect on $5-\mathrm{HT}_{1 \mathrm{~A}}$ receptors $(16)$. As in vertebrates, the $5-\mathrm{HT}_{1}$ agonist produced an inhibitory postsynaptic effect, and the 5- $\mathrm{HT}_{2}$ agonist enhanced the LG neuron's response. However, the serotonin receptors in the stomatogastric nervous system of crustacea have been shown to be quite different from the vertebrate types (17).

Our results show that although many behavior patterns may change quickly during a change in social status, slower changes that affect behavior also occur in the nervous system. The slower change described here is change in the effect of serotonin on the exN citability or threshold of the LG neuron, and thereby on the LG-evoked tailflip behavion The slow rate of this change may result from slow changes in the numbers of different ac tivatable serotonin receptors or from slowס changes in the gains of their different trans. duction pathways. The rate may also be slow⿳亠口冋.

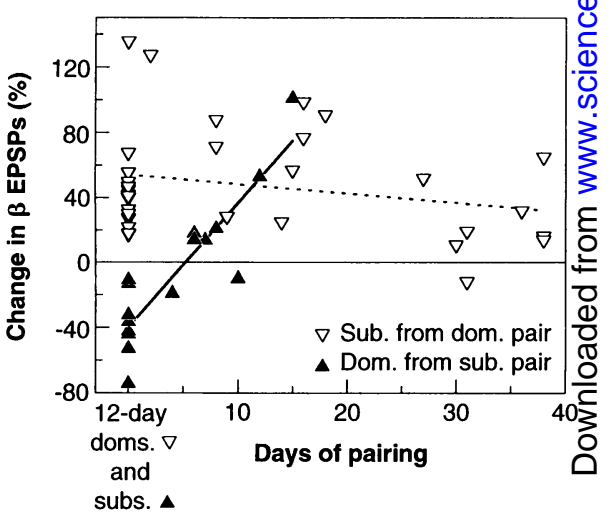

Fig. 5. Effect of social history on serotonin modulation of the LG neuron's response. The change produced by serotonin in $\beta$ EPSPs in the LG neurons of new subordinate crayfish created by pairing of dominant animals for up to 38 days (open inverted triangles), and in $\beta$ EPSPS of new dominant crayfish produced by pairing of subordinate crayfish for up to 15 days (solid triangles) is shown. The leftmost column of points (above 12day doms. and subs.) redisplays serotonininduced changes in EPSPs of 12-day dominant (open inverted triangles) and subordinate (solid triangles) crayfish from the plots in the right and left panels, respectively, of Fig. 4. The dotted line is a linear regression $\left(r^{2}=0.06\right)$ through the open inverted triangles. The slope of the dotted line is $-0.6 \%$ per day, and high and low $95 \%$ confidence limits of the slope are -1.3 and $0.2 \%$ per day, respectively. The heavy solid line is a linear regression $\left(r^{2}=0.78\right)$ through the solid triangles. The slope of the dotted line is $7.6 \%$ per day, and high and low $95 \%$ confidence limits of the slope are 11.6 and $3.7 \%$ per day, respectively. 
because it is adaptive to change 5-HT's effect on the excitability of LG only in response to a persistent change in the animal's social status. In this case, these slow changes in 5-HT's effect could function as part of the "memory" of recent agonist interactions: Too short a memory might needlessly expose the animal to the risks of aggressive interactions that occur between strangers.

We conclude that social experience can modulate neural circuit function by controlling the effect of a neuromodulator on the response of an identified neuron. Presumably this type of neural plasticity mediates the animal's social adaptation by producing experience- and context-dependent changes in the relative excitability of neural circuits. These excitability changes would translate into corresponding changes in the relative frequencies with which different behaviors are expressed. Animals differ in temperament both in their groups and individually over time; it is likely that the type of neuronal and neural circuit changes reported here underlie this sort of behavioral plasticity.

\section{REFERENCES AND NOTES}

1. E. F. Coccaro, Int. Clin. Psychopharmacol. 7, 3 (1992); V. M. Linnoila and M. Virkkunen, J. Clin. Psychiatry 53(suppl.), 46 (1992); S. Winberg, G. E. Nilsson, K. H. Olsen, J. Comp. Physiol. A 170, 93 (1992); H. D. Steklis, M. J. Raleigh, A. Kling, K. Tachiki, Am. J. Primatol. 11, 133 (1986); O. Cases et al., Science 268, 1763 (1995).

2. M. S. Livingstone, R. M. Harris-Warrick, E. A. Kravitz, Science 208, 76 (1980).

3. E. A. Kravitz, ibid. 241, 1775 (1988).

4. D. Glanzman and F. B. Krasne, J. Neurosci. 3, 2263 (1983)

5. F. B. Krasne, J. Exp. Biol. 50, 29 (1969), J. J. Wine and F. B. Krasne, in The Biology of Crustacea, D. C. Sandeman and H. L. Atwood, Eds. (Academic Press, New York, 1982), vol. 4, pp. 241-292.

6. E. T. Vu, S. C. Lee, F. B. Krasne, J. Neurosci. 13 4379 (1993).

7. D. Real and G. Czternasty, Brain Res. 521, 203 (1990)

8. Juvenile ( 1 to $3 \mathrm{~cm}$ ) crayfish (Procambarus clarkii) were hatched and raised in the laboratory, and adult (8 to $10 \mathrm{~cm}$ ) crayfish were obtained commercially. Both were isolated for longer than 1 month. Socially dominant and subordinate animals were created by pairing previously isolated crayfish for 12 days or longer. Juvenile pairs were placed in mesh cages 5 $\mathrm{cm}$ in diameter in groups of eight in 20-gallon aquaria. Adult pairs were placed in individual small aquaria.

9. Crayfish of known dominance status were chilled to immobility, and the abdomen was removed and dissected dorsally to expose the ventral nerve cord. The abdomen was pinned out in a petri dish and maintained in crayfish saline [A. Van Harreveld, Proc. Soc. Exp. Biol. Med. 34, 428 (1936)]. The LG axon was identified visually in the terminal connective and penetrated in the proximal portion of the axon (Fig. 1). Shocks $(0.3 \mathrm{~ms})$ between $3 \mathrm{~V}$ and $7.5 \mathrm{~V}$ were applied to the ipsilateral third and fourth nerves of the terminal ganglion at $90-s$ intervals and evoked both suband superthreshold LG EPSPs. The stimulus series was repeated four times. The preparation was then perfused with serotonin $(100 \mu \mathrm{M}$ in 7 preparations at the outset of these experiments, and $50 \mu \mathrm{M}$ for the remaining 93 preparations) or with a $5-\mathrm{HT}$ agonist ( $50 \mu \mathrm{M}, 67$ preparations), all at $1.2 \mathrm{ml} / \mathrm{min}$. After 45 min of perfusion, responses to four more stimulus series were recorded during continued perfusion. The effects on the LG response produced by the two concentrations of serotonin were indistinguishable. A saline wash followed; responses to a final set of stimuli were recorded after a 1-hour wash. Although the electrophysiology was performed with knowledge of the animals' dominance status, the effects of serotonin on the responses of all three types of animals were unmistakable and immediately distinguishable: The responses of isolate and dominant animals were always greater than those of controls, whereas those of subordinates were always less.

10. S.-R. Yeh, R. A. Fricke, D. H. Edwards, data not shown.

11. D. H. Edwards, W. J. Heitler, E. M. Leise, R. A. Fricke, J. Neurosci. 11, 2117 (1991); R. S. Zucker, J. Neurophysiol. 35, 599 (1972).

12. 5-HT increased EPSPS in the reisolated subordinates (Fig. 4, filled inverted triangles in the left panel) $57.6 \pm 16.5 \%$ (mean \pm SEM) above those of controls, whereas EPSPs in the reisolated dominants (Fig. 4, right panel) were increased $42.1 \pm 14.0 \%$ above those of controls, and EPSPs in isolates (Fig. 4 , left columns of both panels) were $63.3 \pm 8.8 \%$ above those of controls.
13. $5-H T_{1}$ agonist reduced EPSPs in the reisolated subordinates (Fig. 4, open triangles in left panel) $3.7 \pm$ $8.1 \%$ below those of controls, whereas EPSPs in the reisolated dominants (Fig. 4, open triangles in right panel) were increased $3.7 \pm 7.4 \%$ above those of controls, and EPSPS in the isolates (Fig. 4, open triangles in left columns of both panels) were increased $8.4 \pm 10.3 \%$ above those of controls.

14. R. V. Bovbjerg, Physiol. Zool. 26, 173 (1953).

15. S. R. Yeh, R. A. Fricke, D. H. Edwards, unpublished results.

16. R. A. Glennon, J. Med. Chem. 30, 1 (1987); P. P. A Humphrey, P. Haratig, D. Hoyer, Trends Pharmacol. Sci. 14, 233 (1993); B. E. Leonard, Int. Clin. Psychopharmacol. 7, 13 (1992).

17. B. Zhang and R. Harris-Warrick, J. Exp. Biol. 190, 55 (1994)

18. We thank M. Hörner, F. B. Krasne, and E. A. Kravitz for their thoughtful comments on this paper. Supported by NIH research grant RO1 NS26457 and NSF research grant IBN 94-23486.

26 July 1995; accepted 10 November 1995

\title{
Zinc-Induced Collapse of Augmented Inhibition by GABA in a Temporal Lobe Epilepsy Model
}

\author{
Eberhard H. Buhl, Thomas S. Otis, Istvan Mody*
}

In the kindling model of temporal lobe epilepsy, several physiological indicators of inhibition by $\gamma$-aminobutyric acid (GABA) in the hippocampal dentate gyrus are consistent with an augmented, rather than a diminished, inhibition. In brain slices obtained from epileptic (kindled) rats, the excitatory drive onto inhibitory interneurons was increased and was paralleled by a reduction in the presynaptic autoinhibition of GABA release. This augmented inhibition was sensitive to zinc most likely after a molecular reorganization of $\mathrm{GABA}_{\mathrm{A}}$ receptor subunits. Consequently, during seizures, inhibition by GABA may be diminished by the zinc released from aberrantly sprouted mossy fiber terminals of granule cells, which are found in many experimental models of epilepsy and in human temporal lobe epilepsy.

Synaptic inhibition in the mammalian forebrain is primarily mediated by GABA acting at its various receptors $(1,2)$. It is not known by how much the balance between excitation and inhibition has to be offset for pathological changes to occur. A reduced synaptic inhibition will favor hyperexcitability, a condition long associated with epilepsy (3). Although in vitro studies of acute epilepsies have often relied on the experimental impairment of inhibition by GABA (3), the fate of inhibition by GABA in chronic epilepsy models and particularly in human epilepsies remains unclear (3-6). A distinctive sprouting of mossy fibers in the dentate gyrus is shared among human temporal lobe epilepsy (TLE) and several experimental epilepsy models (7-10). The aberrantly sprouted mossy fibers form recurrent

E. H. Buhl, MRC Anatomical Neuropharmacology Unit, Oxford University, Mansfield Road, Oxford OX13TH, UK. T. S. Otis, Department of Neurophysiology, University of Wisconsin Medical School, Medical Sciences Center, 1300 University Avenue, Madison, WI 53706-1532, USA I. Mody, Department of Neurology RNRC 3-131, UCLA School of Medicine, 710 Westwood Plaza, Los Angeles, CA 90095-1769, USA.

*To whom correspondence should be addressed. excitatory synapses with other granule cells but may also contribute to the synaptic drive onto inhibitory interneurons (11). The kindling model of TLE, in which seizures are induced by initially subthreshold electrical stimuli delivered daily to limbic areas over several days or weeks, replicates many anatomical and pathological features, including the sprouting of mossy fibers as seen in human TLE (12). The model is characterized by an enhanced functional inhibition by GABA in the dentate gyrus $(6,13,14)$, but there is no estimate of the excitatory drive onto the inhibitory interneurons.

We measured the degree of glutamatergic excitatory drive onto specific interneurons $(15,16)$ responsible for generating spontaneous inhibitory postsynaptic currents (sIPSCs) in dentate gyrus granule cells after kindling by recording inhibitory currents in the whole-cell configuration (17) at the reversal potential $(0$ to $+5 \mathrm{mV})$ of excitatory synaptic events (18). At this membrane potential, sIPSCs could be detected selectively (Fig. 1A), whereas the excitatory drive onto the interneurons remained intact and could be assessed by the 